\title{
Liposarcoma del cordón espermático simulando una hernia inguinal incarcerada
}

\author{
María Tudela-Lerma ${ }^{1}$, Arturo Colon-Rodríguez ${ }^{1}$, Susana Vázquez ${ }^{1}$, \\ María Isabel Peligros-Gómez y José Luis Porrero-Carro ${ }^{2}$
}

\section{Liposarcoma of the spermatic cord mimicking an incarcerated inguinal hernia}

Introduction: Paratesticular tumors represent 7\%-10\% of intraescrotal masses. Sarcomas account for 90\% of malignant lesions of the spermatic cord and of these approximately 3\%-7\% are liposarcomas. Clinical case: This is the case of a 45 year old male who consulted in the emergency department for a non-reducible right inguino-scrotal mass and was diagnosed with an incarcerated inguinal hernia. Emergency surgery was performed which revealed a large lipomatous tumor, originating from the spermatic cord. Orchiectomy and hernioplasty were performed. Histopathology revealed a well-differentiated liposarcoma of the spermatic cord. Later extension study was conducted, without distant affectation, and did not require adjuvant treatment. Today, after two years of monitoring has been no recurrence. Discussion: Only about 200 cases have been previously reported in the literature and only 61 of these were presented mimicking an incarcerated inguinal hernia. Due to the low incidence of this disease it is difficult to know the natural history and draw conclusions on the results of treatment, which so far remains the radical orchiectomy with wide local excision of the tissue. The role of radiotherapy and chemotherapy is still controversial Key words: liposarcoma; spermatic cord; inguinal hernia.

\section{Resumen}

Introducción: Los tumores paratesticulares representan del 7\%-10\% de las masas intraescrotales. Los sarcomas abarcan el $90 \%$ de las lesiones malignas del cordón espermático y de éstas, aproximadamente, el 3\%-7\% son liposarcomas. Caso clínico: Presentamos el caso de un varón de 45 años, que consultó en urgencias por una masa inguinoescrotal derecha no reductible, diagnosticándose de hernia inguinal incarcerada. Se realizó cirugía urgente evidenciando una gran tumoración de aspecto lipomatoso, dependiente de cordón espermático. Se realizó orquiectomía y hernioplastía inguinal. La anatomía patológica, reveló un liposarcoma bien diferenciado de cordón espermático. Posteriormente, se realizó estudio de extensión, sin afectación a distancia y no precisó tratamiento adyuvante. Actualmente, tras dos años de seguimiento no ha presentado recidiva. Discusión: Sólo alrededor de 200 casos han sido comunicados previamente en la literatura y sólo 61 de éstos se presentaron simulando una hernia inguinal incarcerada. Debido a la baja incidencia de esta patología es difícil de conocer la historia natural y llegar a conclusiones sobre los resultados del tratamiento, el cual hasta el momento sigue siendo la orquiectomía radical, con escisión amplia de los tejidos locales. El papel de la radio y quimioterapia aun es controvertido. Conclusiones: Los sarcomas del cordón espermático son neoplasias raras con alta tasa de recurrencia local. Su manejo inicial es quirúrgico. Se requiere de un alto índice de sospecha clínica para el diagnóstico ya que las implicaciones oncológicas varían en función del tratamiento que, en ocasiones, es llevado a cabo por cirujanos generales al simular una hernia inguinal.

Palabras clave: liposarcoma; cordón espermático; hernia inguinal.
'Hospital Universitario

Gregorio Marañón. Madrid, España.

${ }^{2}$ Hospital Universitario Santa Cristina. Madrid, España.

Recibido el 3 de febrero de 2018 y aceptado para publicación el 3 de junio de 2018

Correspondencia a: Dra. María Tudela-Lerma matuler@hotmail.com 


\section{Introducción}

Los tumores intraescrotales pueden ser testiculares $(90 \%)$ o paratesticulares $(10 \%)$ y de éstos la gran mayoría se concentran en el cordón espermático $(90 \%)^{1}$.

Los tumores paratesticulares malignos representan el 30\%, de éstos la gran mayoría (90\%) son sarcomas, de los cuales el liposarcoma supone el $3 \%-7 \% \%^{2-4}$.

El liposarcoma fue descrito por primera vez en 1952 por Herbert. Es un tumor mesenquimal maligno del tejido adiposo y existen 5 subtipos histológicos ${ }^{5}$ :

- Bien diferenciado

- Mixoide y células redondas

- Pleomórfico

- Lipoblástico

- Fibroblástico

Suelen presentarse en varones de edad media o avanzada y se caracterizan por una masa inguinal o escrotal indolora, por lo que a menudo se confunden con hernias inguinales, hidroceles o incluso epideidimitis crónica ${ }^{6-8}$.

El diagnóstico preoperatorio es difícil y frecuentemente se realiza de forma incidental durante la cirugía9. En caso de sospecha las pruebas complementarias indicadas serían la tomografía computada (TC) o la resonancia magnética $(\mathrm{RM})^{10}$.

El tratamiento recomendado es quirúrgico y consiste en una orquiectomía radical con escisión en bloque amplio de los tejidos circundantes; la resección del hemiescroto no es mandatoria si la piel no

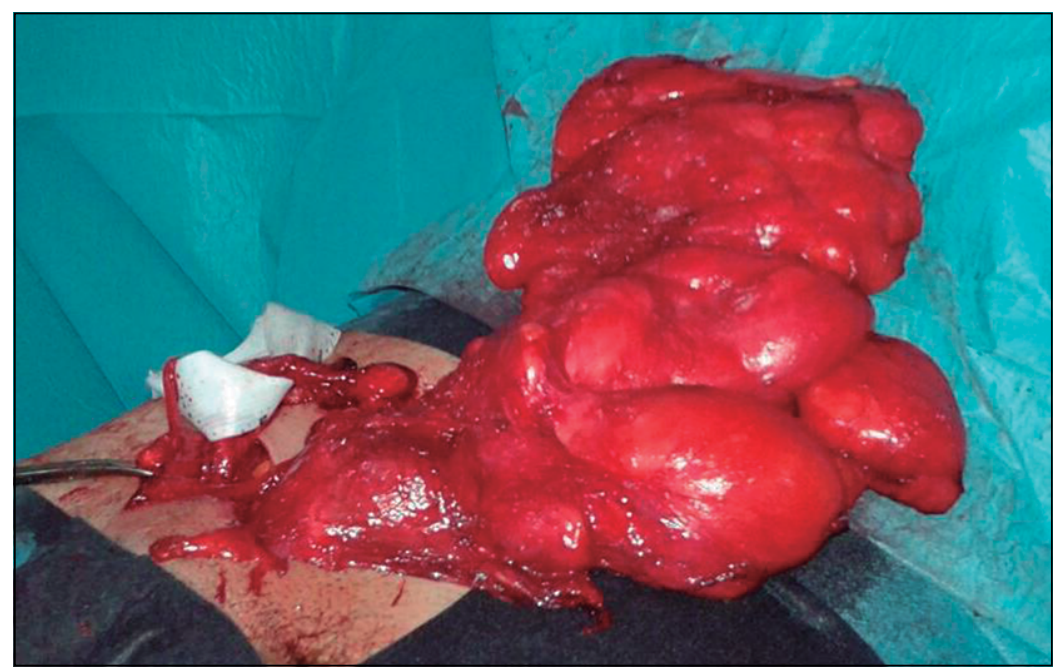

Figura 1. Liposarcoma del cordón espermático. está afectada. El papel de la radio y quimioterapia aun es controvertido. Se recomienda tratamiento adyuvante con radioterapia en casos de márgenes positivos, ganglios positivos o tumores de alto grado. El uso de la quimioterapia adyuvante podría estar indicado en casos de alto grado o metástasis ${ }^{5,11}$.

El patrón de diseminación a nivel local es a través del cordón espermático hacia el canal inguinal y de este a la cavidad abdominal, menos frecuente hematológico y linfático ${ }^{12}$.

Son tumores con alta tasa de recurrencia local, incluso tras varios años de la cirugía, pero con alto índice de supervivencia a los 5 años ${ }^{13-15}$.

\section{Caso clínico}

Presentamos el caso de un varón de 45 años con antecedente de vasectomía, que consultó en urgencias por una masa inguinoescrotal derecha dolorosa de dos días de evolución sin signos de obstrucción intestinal. A la exploración se palpaba una tumoración inguinal derecha dura dolorosa no reductible. Los hallazgos del laboratorio resultaron normales. Con el diagnóstico de hernia inguinal incarcerada, se realizó cirugía urgente evidenciando una gran tumoración $20 \times 15 \times 8 \mathrm{~cm}$ de aspecto lipomatoso, heterogénea, tabicada, dependiente de cordón espermático derecho (Figura 1). Ante la sospecha diagnóstica de un tumor paratesticular maligno, se realizó orquiectomía radical derecha y hernioplastía inguinal urgente. El posoperatorio cursó sin complicaciones. La anatomía patológica, incluyendo pruebas inmunohistoquímicas, resultó un liposarcoma bien diferenciado de cordón espermático (Figura 2). Posteriormente, se realizó estudio de extensión mediante una TC toraco-abdomino-pélvico, que no evidenció afectación a distancia por lo que no precisó tratamiento adyuvante. Actualmente, tras dos años de seguimiento, no ha presentado signos de recidiva local ni aparición de metástasis.

\section{Discusión}

Sólo alrededor de 200 casos de liposarcoma del cordón espermático han sido comunicados previamente en la literatura ${ }^{9}$ y sólo hay recogidos 61 casos cuya forma de presentación fue la simulación de una hernia inguinal incarcerada ${ }^{16}$, como es el caso clínico descrito. Debido a la baja incidencia de esta patología es difícil de conocer la historia natural y llegar a conclusiones sobre los resultados del tratamiento ya que las series más amplias incluyen $10-20$ casos $^{5,17}$. 


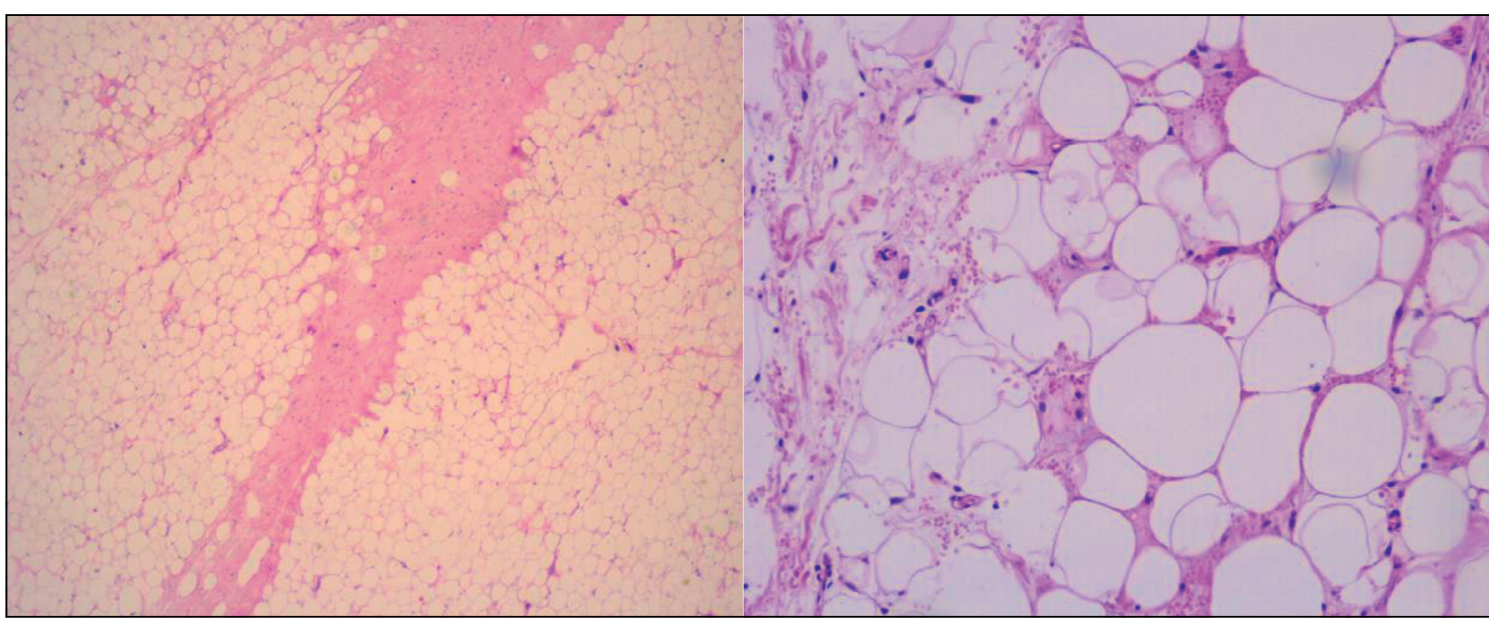

El liposarcoma en esta localización supone el $3 \%$ a $7 \%$ por lo que resulta un subtipo histológico infrecuente $^{2-4}$ (Figura 3). En raras ocasiones los liposarcomas retroperitoneales pueden alcanzar la región inguinal envolviendo al cordón espermático.

Aunque infrecuente, en algunos tumores pueden coexistir varios subtipos simultáneamente, la mayoría de los liposarcomas paratesticulares suelen ser del subtipo bien diferenciado $(40 \%-50 \%)^{18}$, lo cual se correlaciona con el buen pronóstico de estos tumores a pesar del gran tamaño que pueden llegar a alcanzar . Los tumores bien diferenciados, a su vez, se pueden dividir en variante esclerosante (según el contenido de componente fibroso), adipocítico (lipoma-like) -los más frecuentes- así como en inflamatorio o de células con prolongaciones espiculares.

El estudio inmunohistoquímico demuestra que casi todas las células de los liposarcomas son CD34 positivas, el marcador más específico es la proteína
$\mathrm{S} 100$, y los de alto grado suelen expresar la desmina. También pueden haber otros marcadores como la vimentina ${ }^{5,19,20}$. El análisis genético demuestra numerosos protooncogenes siendo para los liposarcomas bien diferenciados el MDM2 y CDK4 en la región cromosómica $12 \mathrm{q} 13-15^{19,21}$.

La edad de presentación más común es entre los 50 y 70 años, el cuadro clínico suele ser una masa escrotal o inguinal palpable en ocasiones acompañado de dolor de leve a moderado o a molestias relacionadas con el tamaño (sensación de pesadez...) incluyéndose como diagnóstico diferencial una hernia inguinal, a veces incluso incarcerada -como es el caso clínico-, hidrocele, epididimitis crónica, lipomas, quistes del cordón o hematocele $e^{6-8,22}$. La duración de la clínica puede ir desde pocos días a años de evolución. Al ser neoplasias de crecimiento lento la tumoración puede alcanzar gran tamaño. La exploración física demuestra una masa sólida irregular con transiluminación negativa.
Figura 2. a. Hematoxilina-eosina $5 X$; b. Hematoxilina-eosina 40X.

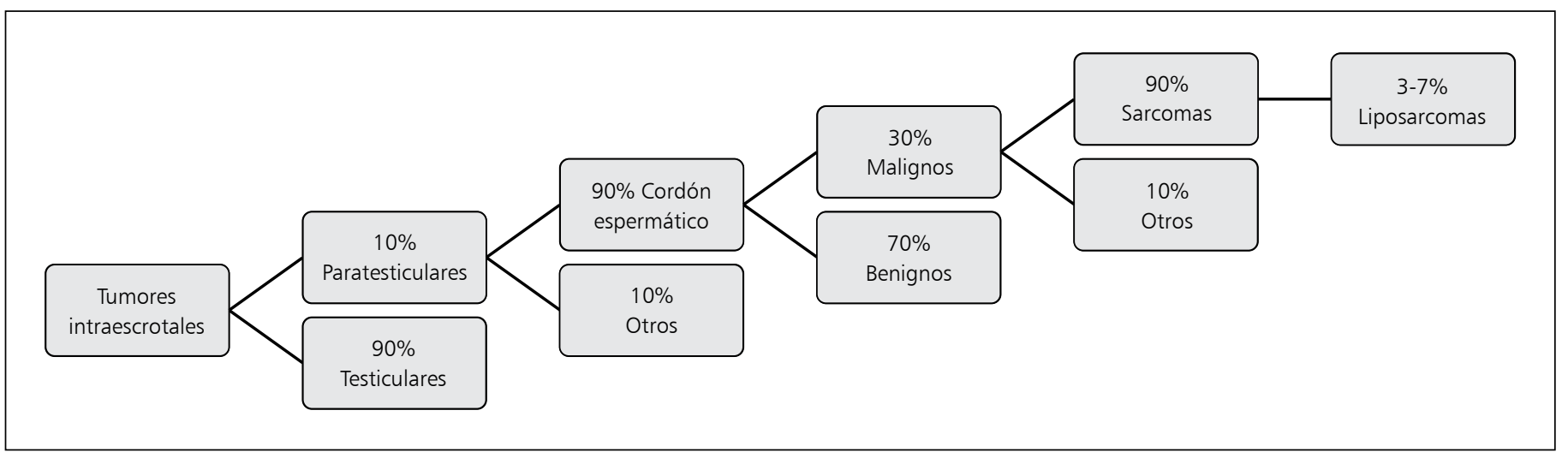

Figura 3. Clasificación Tumores intraescrotales. 
El diagnóstico preoperatorio no es común, lo habitual es un hallazgo incidental intraoperatorio en el contexto de una cirugía realizada al suponer alguna de las patologías que se incluyen en el diagnóstico diferencial; en este caso clínico el diagnóstico fue en el curso de una cirugía urgente realizada por sospecha de hernia inguinal incarcerada ${ }^{23-25}$. Pero en caso de sospecha preoperatoria las pruebas complementarias indicadas serían la TC o la RM. La ecografía no es útil en el caso de los sarcomas. Debido a que no existen características patognomónicas y que la mayoría suelen ser lipomas, la distinción preoperatoria entre tumores benignos y malignos es difícil, pero si se tiene la sospecha diagnóstica la cirugía propuesta debe ser radical ${ }^{22}$.

El tratamiento de esta enfermedad es quirúrgico, se debe realizar una orquiectomía radical con escisión en bloque amplio de tejidos circundantes, no estando indicada la resección del hemiescroto si la piel no está afectada. La linfadenectomía inguinal o retroperitoneal no está indicada, salvo que exista evidencia de metástasis ganglionar, ya que no ofrece beneficio alguno. Esto se explica debido a que el principal patrón de diseminación es local, por contigüidad, a través del cordón espermático hacia el canal inguinal y de este a la cavidad abdominal, menos frecuente hematológico y linfático ${ }^{12}$.

El papel de la radio y quimioterapia aun es controvertido. Se recomienda tratamiento adyuvante con radioterapia en casos de márgenes positivos, ganglios positivos o tumores de alto grado. El uso de la quimioterapia adyuvante podría estar indicado en casos de alto grado o metástasis aunque no hay consenso en la literatura ${ }^{5,11}$.
Son tumores con alta tasa de recurrencia local (55\%-70\%), incluso tras varios años de la cirugía, lo cual obliga a un seguimiento clínico a largo plazo. A pesar de ello el índice de supervivencia a los 5 años es alto $(70 \%)^{13-15,26,27}$.

\section{Conclusiones}

Los liposarcomas del cordón espermático son neoplasias raras, hasta el momento solo hay descritos 61 casos en la literatura. Su manejo inicial es quirúrgico. Se requiere de un alto índice de sospecha clínica para el diagnóstico ya que las implicaciones oncológicas varían en función del tratamiento, que en ocasiones es llevado a cabo por cirujanos generales al simular una hernia inguinal. Debido a que presentan alta tasa de recurrencia local el seguimiento debe ser estrecho y a largo plazo.

\section{Responsabilidades éticas}

Protección de personas y animales. Los autores declaran que para esta investigación no se han realizado experimentos en seres humanos ni en animales.

Confidencialidad de los datos. Los autores declaran que en este artículo no aparecen datos de pacientes.

Derecho a la privacidad y consentimiento informado. Los autores declaran que en este artículo no aparecen datos de pacientes.

\section{Conflictos de interés: No hay.}

\section{Referencias}

1. García Morúa A, Lozano Salina JF, Valdés Sepúlveda F, Zapata H, Gómez Guerra LS. Liposarcoma of the spermatic cord: our experience an review of the literatura. Actas Urológicas Esp. 2009;33:811-5.

2. Panagis A, Karydas G, Vasilakakis J, Chatzipaschalis E, Lambropoulou M, Papadopoulos N. Myxoid liposarcoma of the spermatic cord: A case report and review of the literature. Int Urol Nephrol. 2003;35:369-72.

3. Gómez Dorronsoro ML, Pascual Piedrola I, Cordoba Iturriaga- Goitia A, Valenti Ponsa C, Manrique Celada M, Garrón Aoiz L. Liposarcoma de cordón espermático: criterios de diagnóstico diferencial y tratamiento. Arch Esp Urol. 2000;53:65-7.

4. Fitzgerald S, MacLennan GT. Paratesticular Liposarcoma. J Urol. 2009;181:331-2.

5. Montgomery E, Fisher C. Paratesticular liposarcoma. A clinicopathologic Study. Am J Surg Pathol. 2003;27:40-7.

6. Schwartz SL, Swierzewski SJ, Sondak VK, Grossman HB. Liposarcoma of the spermatic cord: report of 6 cases and review of the literature. J. Urol.1995;153:154-7.

7. Campoy Martínez P, Camacho Martínez E, Arrabal Martín M. Liposarcoma de cordón espermático. un raro tumor urológico. Arch Esp Urol. 1992;45:710.

8. Gago Juan A, Lujan Galán M, Bustamante
Alarma S. Liposarcoma mixoide paratesticular como simulador de proceso herniario. descripción de un caso. Arch. Esp. Urol, 1997;50:921-3.

9. Fubiao Li, Runhui Tian, Changjiu Yin, Xiaofan Dai, Hongliang Wang, Ning Xu, et al. Liposarcoma of the Spermatic Cord Mimicking a Left Inguinal Hernia: A Case Report and Literature Review. World Journal of Surgical Oncology. 2013;11-8.

10. Yang DM, Kim HC, Lim JW, Jin W, Ryu $\mathrm{CW}$, Kim GY, et al. Sonographic findings of groin masses. J Ultrasound Med. 2007;26:605-14.

11. Abolhasani M, Babashahi M, Shooshtarizadeh T, Asgari M, Shahrok $\mathrm{H}$, Shadpour P, et al. Well differentiated liposarcoma of spermatic cord: report of 3 
rare cases. Medical Journal of the Islamic Republic of Iran. 2014;28:10-3.

12. Fagundes M, Zietman A, Althausen A, Coen J, Shipley W. The Management of Spermatic Cord Sarcoma. Cancer 1996;77:1873-6.

13. Gimeno Aránguez M, Escribano Patiño G, Hernández Fernández E. Liposarcomas paratesticulares. Estudio clínico patológico de tres casos y revisión de la literatura. Arch Esp Urol. 2006;59:875-81.

14. Yoshida T, Saito J, Ichimaru N, Miyagawa Y, Nishimura K, Okuyama A, et al. Local recurrence of spermatic cord liposarcoma: a case report. Hinyokika Kiyo 2006; 52:581-4.

15. Hagiwara N, Nishida Y, Fujimoto $Y$, Isogai K, Fujihiro S, Deguchi T. Local Recurrence of Liposarcoma of the spermatic cord 6 years after orchiectomy: a case report. Hinyokika Kiyo 2002;48:443-6.

16. Hassan JM, Quisling SV, Melvin WV, Sharp, KW. Liposarcoma of the espermatic cordomas masquerading as incarcerated inguinal hernia. Am Surg. 2003;69:163-5.
17. Blitzer PH, Dosoretz DE, Proppe KH, Shipley WU. Tratment of malignant tumors of the spermatic cord: a study of 10 cases and review of the literature. J Urol. 1981;126:611-4.

18. Astigueta Pérez A, Pow-Sang Godoy G, Abad Lichman M, Sánchez Lihon J, Morante Deza C, Meza Montoya L, et al. Sarcoma mixto paratesticular: Liposarcoma- Rabdiomiosarcoma. Presentación de un caso y revisión de la literatura. Arch Esp Urol 2007;60:1204-8.

19. Laurino L, Furlanetto A, Orvieto E, Dei Tos A. Well-differentiated Liposarcoma (Atypical Lipomatous Tumors). Semin Diagn Pathol. 2001;18:258-62.

20. Peyri Rey E, Urban Ramon A, Martínez Fernández M, Sanmarti da Silva B. Liposarcoma desdiferenciado de cordón espermático: Degeneración de un lipoma previo resecado. Actas Urol Esp. 2003;27:383-6.

21. Lazarus S, Trombetta L. Ultrastructural and histochemical identification of sclerosing liposarcoma. Histopathology 1981;5:223-35.

22. Bhullar JS, Mohey L, Chaudhary S,
Herschman B, Ferguson L. Liposarcoma of the espermatic cord: an unexpected finding of hernia inguinal repair. Am Surg.2013;79:212-3.

23. Mizuno Y, SumiY, Nachi S, Ito Y, Marui T, Saji S, et al. A case of a large retroperitoneal liposarcoma presenting as incarcerated inguinal hernia. Hernia 2006; 10: 439-42.

24. Hsu YF, Chou YY, Cheng YH. Spermatic cordomyxoid liposarcoma presenting a incarcerated inguinal hernia: report of a case and review of literatura. Hernia 2012;16:719-26.

25. Bognár G, Berecky B, Barabás L, Diczházi C, Onderejka P. Recurrent retroperitoneal liposarcoma presenting as incarcerated femoral hernia. Magy Seb. 2009;62:30-3.

26. Hagiwara N, Nishida Y, Fujimoto Y, Fujihiro S, Deguchi T. Local recurrence of liposarcoma of the espermatic cord after 6 year after orchiectomy: a case report. Hinyokikakiyo 2002;48:443-6.

27. Kryvenko ON, Rosenverg AE, Jorda M, Epstein JI. Differenciated Liposarcoma of the Spermatic Cord: A series of 42 Cases. AM J Surg Pathol. 2015;39:1219-25. 